\title{
Multi-atom quasiparticle scattering interference for superconductor energy-gap symmetry determination
}

\author{
Rahul Sharma ${ }^{1,2,16}$, Andreas Kreisel iD $^{3,16}$, Miguel Antonio Sulangi ${ }^{4,16}$, Jakob Böker ${ }^{5}$, Andrey Kostin ${ }^{1}$, Milan P. Allan ${ }^{6}, \mathrm{H}$. Eisaki ${ }^{7}$,
} Anna E. Böhmer ${ }^{8,9}$, Paul C. Canfield ${ }^{9,10}$, Ilya Eremin ${ }^{5,11}$, J. C. Séamus Davis $\mathbb{D}^{1,12,13,14 凶}$, P. J. Hirschfeld ${ }^{4}$ and Peter O. Sprau $^{1,15}$

Complete theoretical understanding of the most complex superconductors requires a detailed knowledge of the symmetry of the superconducting energy-gap $\Delta_{\mathbf{k}}^{a}$, for all momenta $\mathbf{k}$ on the Fermi surface of every band $a$. While there are a variety of techniques for determining $\left|\Delta_{\mathbf{k}}^{a}\right|$, no general method existed to measure the signed values of $\Delta_{\mathbf{k}}^{a}$. Recently, however, a technique based on phaseresolved visualization of superconducting quasiparticle interference (QPI) patterns, centered on a single non-magnetic impurity atom, was introduced. In principle, energy-resolved and phase-resolved Fourier analysis of these images identifies wavevectors connecting all $\mathbf{k}$-space regions where $\Delta_{\mathbf{k}}^{a}$ has the same or opposite sign. But use of a single isolated impurity atom, from whose precise location the spatial phase of the scattering interference pattern must be measured, is technically difficult. Here we introduce a generalization of this approach for use with multiple impurity atoms, and demonstrate its validity by comparing the $\Delta_{\mathbf{k}}^{a}$ it generates to the $\Delta_{\mathbf{k}}^{a}$ determined from single-atom scattering in FeSe where $s_{ \pm}$energy-gap symmetry is established. Finally, to exemplify utility, we use the multi-atom technique on LiFeAs and find scattering interference between the hole-like and electronlike pockets as predicted for $\Delta_{\mathbf{k}}^{a}$ of opposite sign.

npj Quantum Materials (2021)6:7; https://doi.org/10.1038/s41535-020-00303-4

\section{INTRODUCTION}

The macroscopic quantum condensate of electron pairs in a superconductor is represented by its order-parameter $\Delta_{\mathbf{k}}^{a} \propto\left\langle c_{\mathbf{k}}^{a \dagger} c_{-\mathbf{k}}^{a \dagger}\right\rangle$, where $c_{\mathbf{k}}^{a \dagger}$ is the creation operator for an electron with momentum $\mathbf{k}$ on band $a$. But electron pair formation can occur through a wide variety of different mechanisms and in states with many possible symmetries ${ }^{1}$. Thus, it is the symmetry properties of $\Delta_{\mathbf{k}}^{a}$ that are critical for identification of the Cooper pairing mechanism ${ }^{1}$ and, moreover, for understanding the macroscopic phenomenology ${ }^{1}$. While macroscopic techniques can reveal energy-gap symmetry for single-band systems ${ }^{2,3}$, no general technique existed to determine the relative signs of $\Delta_{\mathbf{k}}^{a}$ and $\Delta_{\mathbf{k}^{\prime}}^{\beta}$ between $\mathbf{k}_{a}$ and $\mathbf{k}_{\beta}$ for all Fermi surface (FS) momenta in an arbitrary superconductor.

In 2015, a conceptually simple and powerful technique for determining $\Delta_{\mathbf{k}}^{a}$ symmetry was introduced ${ }^{4}$, by Hirschfeld, Eremin, Altenfeld, and Mazin (HAEM). It is based on interference of weakly scattered quasiparticles at a single, non-magnetic, impurity atom. Given a superconductor Hamiltonian

$\mathcal{H}_{\mathbf{k}}=\left(\begin{array}{cc}H_{\mathbf{k}} & \Delta_{\mathbf{k}} \\ \Delta_{\mathbf{k}}^{\dagger} & -H_{-\mathbf{k}}^{T}\end{array}\right)$

where $H_{\mathbf{k}}$ is the normal-state Hamiltonian and $\Delta_{\mathbf{k}}$ the superconducting energy gap, a non-magnetic impurity atom is modeled as a weak point-like potential scatterer, with Hamiltonian $H_{\text {imp }}=V_{0} c_{\mathbf{r}}^{\dagger} c_{\mathbf{r}}$ centered at the origin of coordinates $\mathbf{r}=0$. Effects of scattering are then represented by a T-matrix derived from the local Green's function $G_{0}(E)=\sum_{\mathbf{k}} G_{\mathbf{k}}^{0}(E)$, where $G_{\mathbf{k}}^{0}(E)=\left(E+i 0^{+}-\mathcal{H}_{\mathbf{k}}\right)^{-1}$. When the impurity potential is constant in k-space, the Green's function becomes $G_{\mathbf{k}, \mathbf{k}^{\prime}}(E)=G_{\mathbf{k}, \mathbf{k}^{\prime}}^{0}(E)+G_{\mathbf{k}}^{0}(E) T(E) G_{\mathbf{k}^{\prime}}^{0}(E)$, with the T-matrix given by $T(E)=\left[1-V_{\mathrm{imp}} G_{0}(E)\right]^{-1} V_{\mathrm{imp}}$, where $V_{\mathrm{imp}}$ is the impurity matrix. From $G_{\mathbf{k}, \mathbf{k}^{\prime}}(E)$, the perturbations to the local density-of-states $\delta N(\mathbf{r}, E)$ are predicted surrounding the impurity atom, and its Fourier transform can be determined directly from $\Delta_{\mathbf{k}}$ as

$$
\delta N(\mathbf{q}, E)=-\frac{1}{\pi} \operatorname{Im}\left[\sum_{\mathbf{k}} G_{\mathbf{k}}^{0}(E) T(\omega) G_{\mathbf{k}+\mathbf{q}}^{0}(E)\right]_{11},
$$

which is a purely real quantity because, in the theoretical calculation, the single impurity is exactly at the origin of coordinates. The authors of ref. 4 realized that the particle-hole symmetry of Eq. (2) for scattering interference wavevector $\mathbf{q}=\mathbf{k}_{\mathrm{f}}^{\beta}-\mathbf{k}_{\mathrm{i}}^{\alpha}$, depends on the relative sign of the energy-gaps $\Delta_{\mathbf{k}_{\mathbf{i}}}^{a}$ and $\Delta_{\mathbf{k}_{\mathbf{f}}}^{\beta}$ at these two momenta. Consequently, the experimentally accessible energy-antisymmetrized function $\rho^{-}(\mathbf{q}, E)$ of phase-resolved Bogoliubov scattering interference amplitudes

$$
\rho^{-}(\mathbf{q}, E) \equiv \operatorname{Re}\{\delta N(\mathbf{q},+E)-\delta N(\mathbf{q},-E)\}
$$

can be used to determine the relative sign of the superconducting energy-gaps connected by $\mathbf{q}=\mathbf{k}_{\mathrm{f}}^{\beta}-\mathbf{k}_{\mathrm{i}}^{\alpha}$. In the

\footnotetext{
'Department of Physics, Cornell University, Ithaca, NY 14850, USA. ${ }^{2}$ Maryland Quantum Materials Center, Department of Physics, University of Maryland, College Park, MD 20742 , USA. ${ }^{3}$ Institut für Theoretische Physik, Universität Leipzig, Brüderstr. 16, 04103 Leipzig, Germany. ${ }^{4}$ Department of Physics, University of Florida, 2001 Museum Rd, Gainesville, FL 32611, USA. ${ }^{5}$ Institut für Theoretische Physik, Ruhr-Universität Bochum, D-44801 Bochum, Germany. ${ }^{6}$ Leiden Institute of Physics, Leiden University, Niels Bohrweg 2, 2333 CA Leiden, The Netherlands. ${ }^{7}$ National Inst. of Advanced Industrial Science and Tech., Tsukuba, Ibaraki 305-8568, Japan. ${ }^{8}$ Institute for Quantum Materials and Technologies, Karlsruhe Institute of Technology (KIT), 76133 Karlsruhe, Germany. ${ }^{9}$ Ames Laboratory, U.S. Department of Energy, Ames, IA 50011, USA. ${ }^{10}$ Department of Physics, lowa State University, Ames, IA 50011, USA. ${ }^{11}$ Institute of Physics, Kazan Federal University, Kazan 420008, Russian Federation. ${ }^{12}$ Department of Physics, University College Cork, Cork T12 R5C, Ireland. ${ }^{13}$ Max-Planck Institute for Chemical Physics of Solids, D-01187 Dresden, Germany. ${ }^{14}$ Clarendon Laboratory, University of Oxford, Oxford OX1 3PU, UK. ${ }^{15}$ Advanced Development Center, ASML, Wilton, CT 06897, USA. ${ }^{16}$ These authors contributed equally: Rahul Sharma, Andreas Kreisel, Miguel Antonio Sulangi. ${ }^{\bowtie}$ email: jcseamusdavis@gmail.com
} 

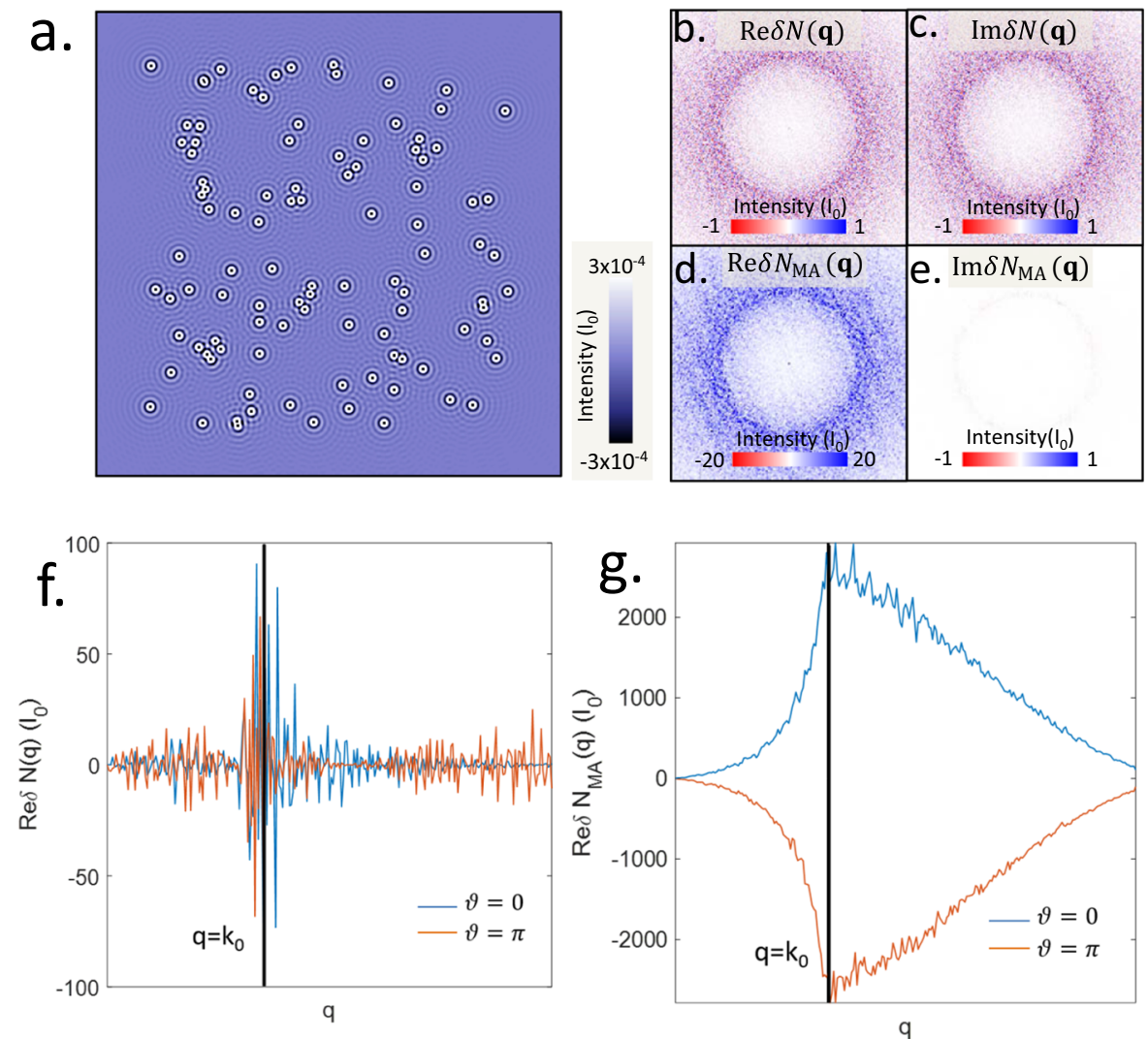

Fig. 1 Schematic for multi-atom phase analysis. a Simulation of density of states perturbation $\delta N(r)$ in amplitude units $I_{0}$ due to twodimensional Friedel oscillations surrounding 100 impurity atoms at random locations $\mathbf{R}_{i}$. b Real part of Fourier transform Re $\delta N(\mathbf{q})$ from $\delta N(\mathbf{r})$ in a. We use an integer grid, hence the units of Fourier transform are also $I_{0}$. c Imaginary part of Fourier transform Re $\delta N(q)$ from $\delta N(r)$ in a. $\mathbf{d}$ Real part of Fourier transform $\operatorname{Re} \delta N_{\text {MA }}(\mathbf{q})$ calculated using multi-atom technique of Eq. (7). e Imaginary part of Fourier transform Re $\delta N_{\text {MA }}(\mathbf{q})$ calculated using multi-atom technique of Eq. (7). $\mathbf{f} \operatorname{Re} \delta N(\mathbf{q})$ from $\delta N(\mathbf{r})$ in a for $\vartheta=0$ and $\vartheta=\pi$, integrated azimuthally from $\mathbf{b}$. Its strong random fluctuations versus $|\mathbf{q}|$ are due to summing the Friedel oscillations in $\delta N(\mathbf{r})$ of a with random phases due to the random locations $\mathbf{R}_{i} . \mathbf{g}$ $\operatorname{Re} \delta N_{\text {MA }}(\mathbf{q})$ from $\delta N(\mathbf{r})$ in a integrated azimuthally from $\mathbf{d}$. $\operatorname{Re} \delta N_{\mathrm{MA}}(\mathbf{q})$ is now orders of magnitude more intense than in $\mathbf{f}$, and the phase of the Friedel oscillations in $\delta N(\mathbf{r})$ of $\mathbf{a}$ is now very well defined because the effects of random locations $\mathbf{R}_{i}$ are removed by using Eq. (7). Note that, now, changing the oscillation phase $\vartheta=0$ to $\vartheta=\pi$ surrounding all $\mathbf{R}_{i}$ in $\delta N(\mathbf{r})$ produces the correct evolution of $\operatorname{Re} \delta N_{\mathrm{MA}}(\mathbf{q})$.

simplest case with two isotropic gaps $\Delta^{\alpha}$ and $\Delta^{\beta}$ on distinct bands, it was demonstrated that

$\rho^{-}(\mathbf{q}, E) \propto \operatorname{Im}\left[\left(E_{+}^{2}-\Delta^{a} \Delta^{\beta}\right) / \sqrt{E_{+}^{2}-\left(\Delta^{\alpha}\right)^{2}} \sqrt{E_{+}^{2}-\left(\Delta^{\beta}\right)^{2}}\right]$

where $E_{+}=E+i 0^{+}$, so that the functional form of $\rho^{-}(\mathbf{q}, E)$ is very different when the product $\Delta^{a} \Delta^{\beta}$ is positive or negative. An elementary implication of Eq. (4) is that, when the order parameter has opposite signs on the two bands so that $\Delta^{a} \Delta^{\beta}<$ $0, \rho^{-}(\mathbf{q}, E)$ does not change sign and exhibits pronounced maxima or minima near $E \approx \Delta^{a, \beta}$ whereas if the order parameter has the same sign so that $\Delta^{a} \Delta^{\beta}>0, \rho^{-}(\mathbf{q}, E)$ exhibits weak maxima or minima near $E \approx \Delta^{\alpha, \beta}$ with a sign of change in between. More generally, especially with multiple bands and anisotropic gaps, HAEM requires that $\rho^{-}(\mathbf{q}, E)$ be predicted in detail for a specific $H_{\mathbf{k}}$ and $\Delta_{\mathbf{k}}$ in Eq. (1) and then compared with quasiparticle interference imaging ${ }^{5}$ in which the scanning tunneling microscope (STM) differential electron tunneling conductance, $g(\mathbf{r}, E) \propto \delta N(\mathbf{r}, E)$ is visualized.

This single-atom phase-resolved HAEM method has been established experimentally ${ }^{6,7}$. For example, in the case of the multiband $s_{ \pm}$superconductor FeSe, the complete energy and wavevector dependence of $\rho^{-}(\mathbf{q}, E)$ was used to determine that the $\mathbf{k}$-space structure including relative sign of $\Delta_{\mathbf{k}}^{a}$ and $\Delta_{\mathbf{k}^{\prime}}^{\beta}$ for all $\mathbf{k}_{\alpha}$ and all $\mathbf{k}_{\beta}$ on two different bands. But this result required that the impurity atom be highly isolated from other impurities and centered precisely at the origin of coordinates, with respect to which the $\operatorname{Re} \delta N(\mathbf{q}, E)$ of Eq. (3) is then properly defined. This was critical because, an error of on the order of $\sim 1 \%$ of a crystal unit cell in the coordinate of the origin (at the impurity atom) produces significant errors in $\operatorname{Re} \delta N(\mathbf{q}, E)$ and $\operatorname{Im} \delta N(\mathbf{q}, E)$ (Supplementary Note 1 and Fig. S1). Moreover, single impurity atom-based measurements limit the $\mathbf{k}$-space resolution because the field of view (FOV) is typically restricted in size, making them unsuitable for superconductors with large impurity-atom densities. This provides the motivation for a variety of approaches to $\Delta_{\mathbf{k}}^{a}$ determination beyond single-atom HAEM. One is to study Bogoliubov bound-states at individual impurity atoms $s^{8-10}$, although this has proven problematic because the elementary HAEM concept (Eq. (3)) is only valid in the weak scattering range, i.e. well below the scattering strength sufficient to generate Bogoliubov bound states ${ }^{11}$. Another approach is to use sparse blind deconvolution ${ }^{12}$ to analyze images of scattering interference at multiple atoms, yielding the phase-resolved real space structure of $\delta N(\mathbf{r}, E)$ although not the $\rho^{-}(\mathbf{q}, E)$ of Eq. (3). Overall, therefore, widespread application of the HAEM technique (Eq. (3)) as a general tool for $\Delta_{\mathbf{k}}^{a}$ determination remains a challenge.

Here, we introduce a practical technique for determining $\rho^{-}(\mathbf{q}, E)$ of Eq. (3) from multiple impurity atoms in a large FOV. To understand this approach, consider the key issue of phase analysis as depicted in Fig. 1, a schematic simulation of Friedel oscillations $\delta N(\mathbf{r})=I_{0} \sum_{\mathbf{R}_{i}} \cos \left(2 \mathbf{k}_{\mathrm{F}} \cdot\left(\mathbf{r}-\mathbf{R}_{i}\right)+\vartheta\right) /\left|\mathbf{r}-\mathbf{R}_{i}\right|^{2}$ from multiple 
a.

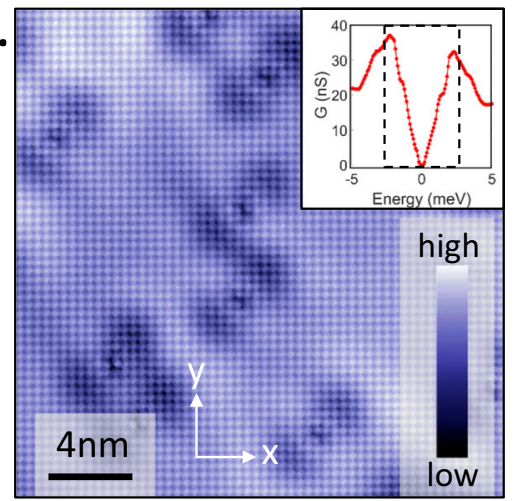

C.

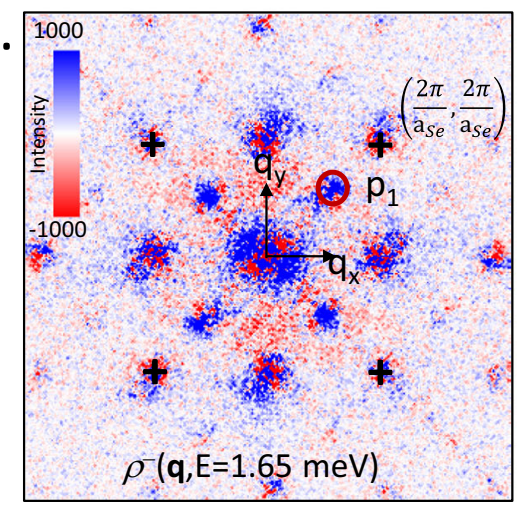

b.
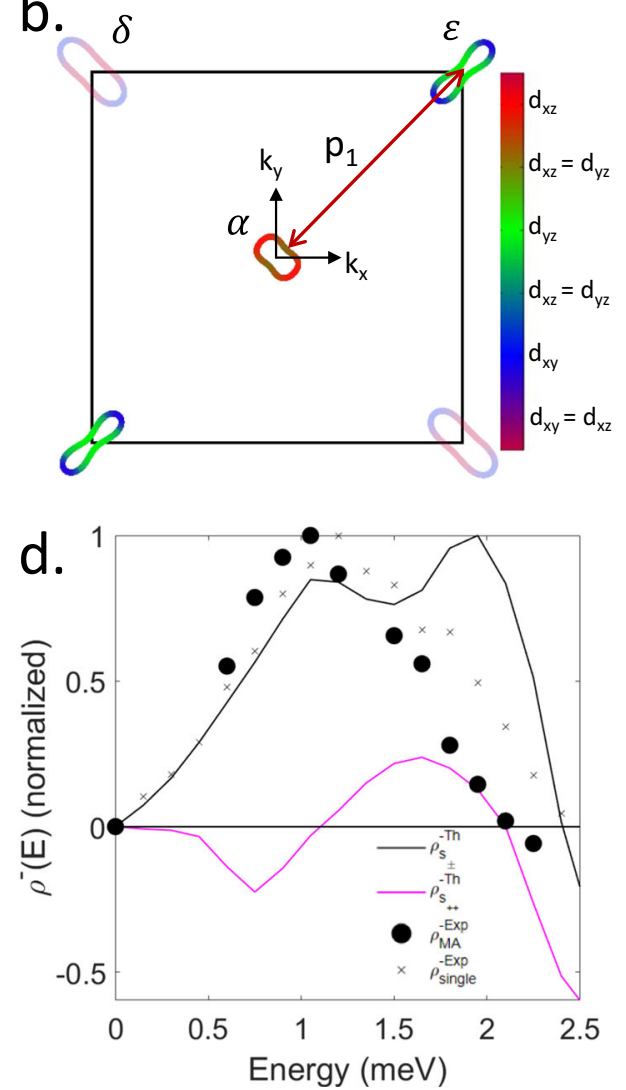

Fig. 2 Demonstration of MAHAEM for FeSe. a Topography of FeSe showing the type of defects (Fe-atom vacancies) used for analysis. The $x$ and $y$-axes are along top Se-Se atoms. Inset shows the differential conductance spectrum recorded at a point on superconducting FeSe. The dashed rectangle represents the energy limits of the high-resolution $\mathrm{d} / / \mathrm{d} V$ maps used herein for $\rho^{-}(\mathbf{q}, E)$ analysis. b Fermi surface of FeSe showing the scattering between hole-pocket $a$ and electron-pocket $\varepsilon$ with scattering vector $\mathbf{p}_{1}$, which is the subject of study. The delta pocket is predicted in LDA calculations, hence it is shown dim in the image, but is now reported not to exist in reality ${ }^{6,38}$. Due to the orbital content, the scattering between quasi-parallel Fermi surfaces would be strongly suppressed in this orbitally selective material ${ }^{6}$. $\mathbf{c} \rho_{\mathrm{MA}}^{-}\left(\mathbf{q}, E=1.05 \mathrm{meV}^{\prime}\right)$ calculated using Eq. (9) for a FOV containing 17 Fe-vacancies. The circle denotes the region where the $a \rightarrow \varepsilon$ scattering occurs and we integrate the $\rho_{\mathrm{MA}}^{-}(\mathbf{q}, E)$ over the $\mathbf{q}$ in this region. Black crosses denote the Bragg peaks. d The integrated $\rho_{\mathrm{MA}}^{-}(E)$ (dots, black) from our MAHAEM analysis of FeSe compare to the theoretical predictions from an accurate band- and gap-structure model of FeSe for $\mathrm{s}_{++}$(solid, pink) and $s_{ \pm}$(solid, black) superconducting energy gap symmetry, and to measured $\rho_{\text {single }}^{\text {-Exp }}(E)$ (crosses, black) from single impurity analysis as reported in ref. ${ }^{6}$. Clearly, the single atom $\rho_{\text {single }}^{- \text {Exp }}(E)$ and the MAHAEM $\rho_{\mathrm{MA}}^{-}(E)$ are in good agreement. Note that the $2 \mathrm{D}$ plots may show both red and blue colors due to non-ideal nature of real experimental data. However, subsequent to the integration over relevant q-space region, the $\rho_{\mathrm{MA}}^{-}(E)$ is well defined as demonstrated here.

atoms at random locations $\mathbf{R}_{\text {i }}$. The Fourier transform components of this $\delta N(\mathbf{r})$ are shown in the top two panels of Fig. $1 \mathrm{~b}$. Obviously, the $\operatorname{Re} \delta N(\mathbf{q})$ required for the HAEM technique in Eq. (3), is weak, does not have a clear sign, and is indistinguishable from $\operatorname{Im} \delta N(\mathbf{q})$. Such effects occur because the spatial phases of all the individual Friedel oscillations at $\mathbf{R}_{i}$ are being added at random. The consequence is most obvious in the azimuthally integrated $\operatorname{Re} \delta N(\mathbf{q})$ shown in Fig. If where the phase information of single-atom Friedel oscillation is completely scrambled and the HAEM technique of Eq. (3) thereby rendered inoperable.

This problem could be mitigated if the Fourier transform of the scattering interference pattern surrounding each $\mathbf{R}_{i}$ were evaluated as if it were at the zero of coordinates. In this regard, consider the Fourier transform of a scattering interference surrounding a single impurity atom at $\mathbf{R}_{i}=\left(x_{i}, y_{i}\right)$,

$$
\int \delta N\left(\mathbf{r}-\mathbf{R}_{i}\right) \mathrm{e}^{\mathrm{i} \mathbf{q} \cdot \mathbf{r}} d r=\mathrm{e}^{\mathrm{i} \mathbf{q} \cdot \mathbf{R}_{i}} \int \delta N\left(\mathbf{r}-\mathbf{R}_{i}\right) \mathrm{e}^{\mathrm{i} \mathbf{q} \cdot\left(\mathbf{r}-\mathbf{R}_{i}\right)} d\left(\mathbf{r}-\mathbf{R}_{i}\right)=\mathrm{e}^{\mathrm{i} \mathbf{q} \cdot \mathbf{R}_{i}} \delta N(\mathbf{q}) .
$$

This "shift theorem" shows how the correctly phase-resolved Fourier transform of a $\delta N_{i}(\mathbf{r})$ oscillation centered on an atom located at $\mathbf{R}_{i}=\left(x_{i}, y_{i}\right)$, can be determined using

$$
\delta N_{i}(\mathbf{q})=\mathrm{e}^{\mathrm{i} \mathbf{q} \cdot \mathbf{R}_{i}} \delta N(\mathbf{q}),
$$

where $\delta N(\mathbf{q})$ is the Fourier transform using the same arbitrary origin as determines the $\mathbf{R}_{\boldsymbol{i}}$. Thus we may define a multi-atom phase-preserving algorithm for QPI

$$
\delta N_{\mathrm{MA}}(\mathbf{q})=\sum_{\mathbf{R}_{i}} \delta N_{i}(\mathbf{q})=\delta N(\mathbf{q}) \sum_{\mathbf{R}_{i}} \mathrm{e}^{\mathrm{i} \mathbf{q} \cdot \mathbf{R}_{i}} .
$$

The consequences of Eq. (7) are illustrated in Fig. 1d, e (Supplementary Note 3 and Supplementary Fig. S4). The real part $\operatorname{Re} \delta N_{\text {MA }}(\mathbf{q})$ now becomes well-defined and the overall magnitude is also strongly enhanced compared to $\operatorname{Re} \delta N(\mathbf{q})$. Moreover, the azimuthally integrated $\operatorname{Re} \delta N_{\text {MA }}(\mathbf{q})$ plotted in Fig. $1 \mathrm{~g}$ shows that the sign of $\operatorname{Re} \delta N_{\mathrm{MA}}(\mathbf{q})$ changes for $\vartheta=0$ and $\vartheta=\pi$ as expected. Here it is essential that the impurity atom coordinates $\mathbf{R}_{i}$ be determined accurately so that the phase is well-defined. We therefore employ a picometer-scale transformation ${ }^{13-15}$ which renders topographic images $T(\mathbf{r})$ perfectly periodic with the lattice, and then use the same transformation on the simultaneously 

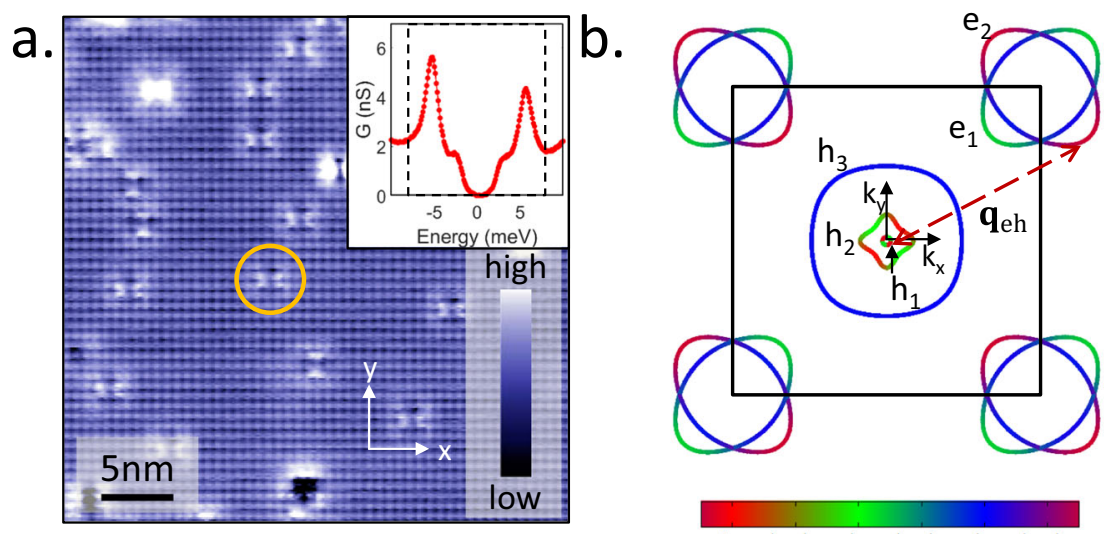

C.

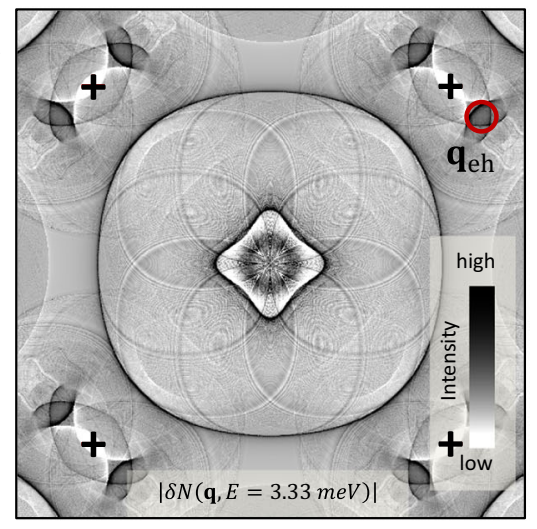

d.

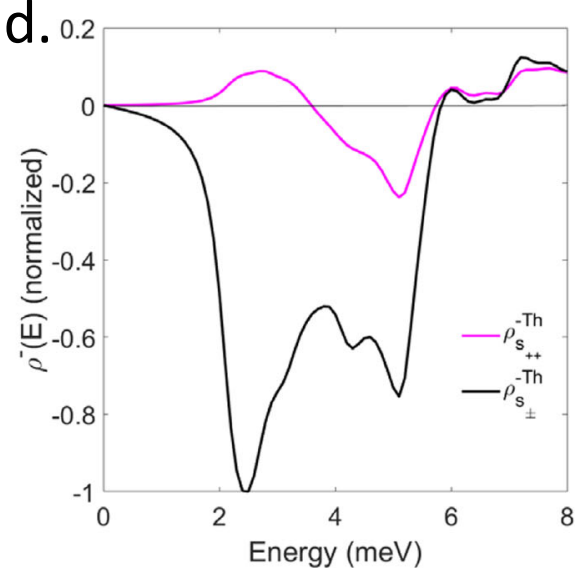

Fig. 3 LiFeAs scattering interference. a Topograph recorded at a LiFeAs surface showing Fe-atom vacancies. The $x$-axis and the $y$-axis directions are along As-As directions. The orange circle denotes the type of weak scatterer we chose for our MAHAEM analysis. Inset shows the $g(E)$ spectrum measured at a point for superconducting LiFeAs. The dashed rectangle represents the energy limits of the high-resolution $\mathrm{d} / / \mathrm{d} V$ maps used herein for $\rho^{-}(\mathbf{q}, E)$ analysis. b The Fermi surface model for LiFeAs showing three hole pockets $h_{1}, h_{2}$, and $h_{3}$ around $\Gamma$-point and two electron pockets $\mathrm{e}_{1}$ and $\mathrm{e}_{2}$ around X-point in a 2-Fe zone. The scattering from hole-like to electron-like pockets takes place as indicated by a dashed vector $\mathbf{q}_{\text {eh. }} \mathbf{c}$ Theoretical prediction for single atom $|\delta N(\mathbf{q}, E=3.25 \mathrm{meV})|$ using band-and gap-structure values fitted from experiments ${ }^{18}$. The electron-hole scattering near $\mathbf{q}_{\mathrm{eh}}$ appears as a "horn"-shaped feature which is enclosed by a circle. $\mathbf{d}$ Theoretical prediction for single atom $\rho^{-}(E)$ integrated over the circular region shown in Fig. $3 c$ for both $s_{ \pm}$(black) and $s_{++}$(pink) symmetry.

recorded $g(\mathbf{r}, E)$ to register all the scattering interference oscillations precisely to the crystal lattice (Supplementary Note 2).

Equation 7 then allows to correctly define the quantities in Eq. (3) for arbitrarily large numbers of scattering atoms. By using the analog of Eq. (6) for $g(\mathbf{r}, E) \propto \delta N(\mathbf{r}, E), \rho^{-}(\mathbf{q}, E)$ for each impurity atom is determined from

$\rho_{i}^{-}(\mathbf{q}, E) \propto \operatorname{Re}\left\{g(\mathbf{q},+E) \mathrm{e}^{\mathrm{i} \mathbf{q} \cdot \mathbf{R}_{i}}\right\}-\operatorname{Re}\left\{g(\mathbf{q},-E) \mathrm{e}^{\mathrm{i} \mathbf{q} \cdot \mathbf{R}_{i}}\right\}$,

while from Eq. (7) the sum over these $\rho_{i}^{-}(\mathbf{q}, E)$ yields

$\rho_{\mathrm{MA}}^{-}(\mathbf{q}, E)=\sum_{i} \rho_{i}^{-}(\mathbf{q}, E)$.

This procedure adds all the individual $\rho_{i}^{-}(\mathbf{q}, E)$ signals from every impurity atom at $\mathbf{R}_{i}$ in-phase, while effectively averaging out the random phase variations due to both locating the origin and the contributions of all other scatterers (Supplementary Fig. S5). We designate this procedure multi-atom HAEM (MAHAEM).

\section{RESULTS AND DISCUSSIONS}

Multi-atom quasiparticle interference for $\Delta_{\mathbf{k}}^{a}$ determination

Determination of the magnitude of superconducting energy gaps $\left|\Delta_{k}^{a}\right|$ has long been achieved ${ }^{16-23}$ using quasiparticle scattering interference (QPI). MAHAEM is the most recent advance of the QPI technique, and to test it we consider FeSe where the single impurity atom HAEM technique for determining $\Delta_{\mathbf{k}}^{a}$ was established experimentally ${ }^{6}$. We measure the differential tunneling conductance $g(\mathbf{r}, E) \equiv \mathrm{d} l / \mathrm{d} V(\mathbf{r}, E)$ in a $30 \mathrm{~nm}$ FOV at $T=$ $280 \mathrm{mK}$, followed by determination of $\mathbf{R}_{i}=\left(x_{i}, y_{i}\right)$ for 17 scattering sites (Supplementary Note 3), some of which are shown in the FOV in Fig. 2a (Supplementary Fig. S2 shows all the sites). These sites are well-known Fe-atom vacancies identified by their crystal locations, and are non-magnetic ${ }^{6}$; their empirical identicality is confirmed by high-resolution electronic structure imaging. We then use Eq. (9) to calculate $\rho_{\overline{M A}}^{-}(\mathbf{q}, E)$. Figure $2 b$ shows the FeSe FS with the hole-pocket $a$ around $\Gamma$-point and electron pockets $\varepsilon$ $(\delta)$ around $X(Y)$ points. Scattering between $a$ and $\varepsilon$ at wavevector $\mathbf{p}_{1}$ was studied. A representative layer $\rho_{\mathrm{MA}}^{-}(\mathbf{q}, E=1.05 \mathrm{meV})$ is shown in Fig. $2 c$, where the scattering feature at vector $\mathbf{p}_{1}$ is marked with a circle. We then sum over the encircled $\mathbf{q}$-region to get $\rho_{\mathrm{MA}}^{-}(E)$ for this scattering feature which is shown as black dots in Fig. 2d. Results from our MAHAEM measurements agree very well with the experimental results using a single impurity

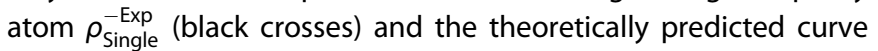
for $\rho_{\mathrm{s}_{ \pm}}^{- \text {Th }}$ (solid, black) in FeSe. This demonstrates the validity and utility of the multi-atom HAEM technique.

Next we consider LiFeAs, a complex iron-based superconductor that is a focus of contemporary physics interest ${ }^{24-26}$, particularly the relative sign of $\Delta_{\mathbf{k}}^{a}$ between all five bands. Figure $3 \mathrm{~b}$ shows the FS of LiFeAs calculated using a tight-binding fit ${ }^{27,28}$ to the experimental data. It consists of three hole pockets $h_{1}, h_{2}$, and $h_{3}$ 
a.
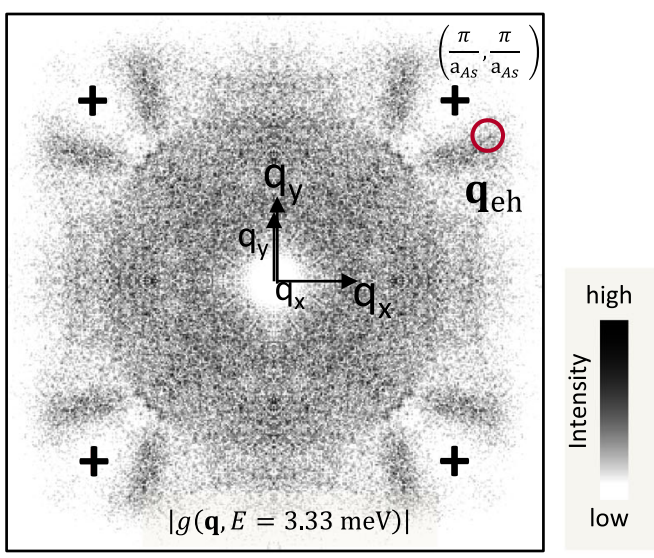

b.
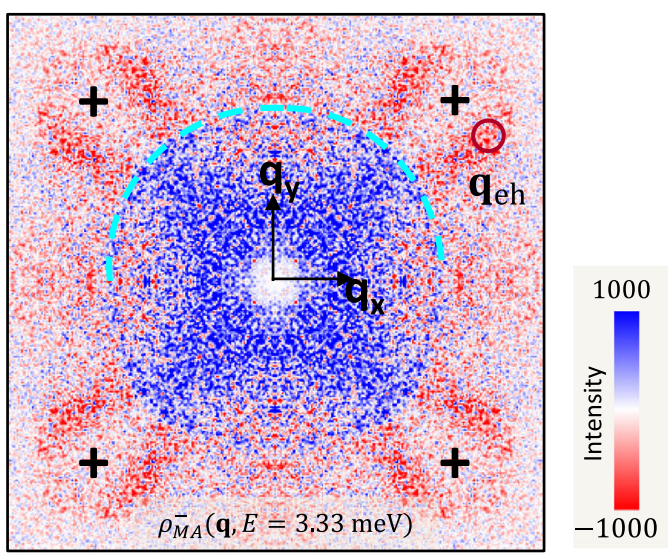

C.

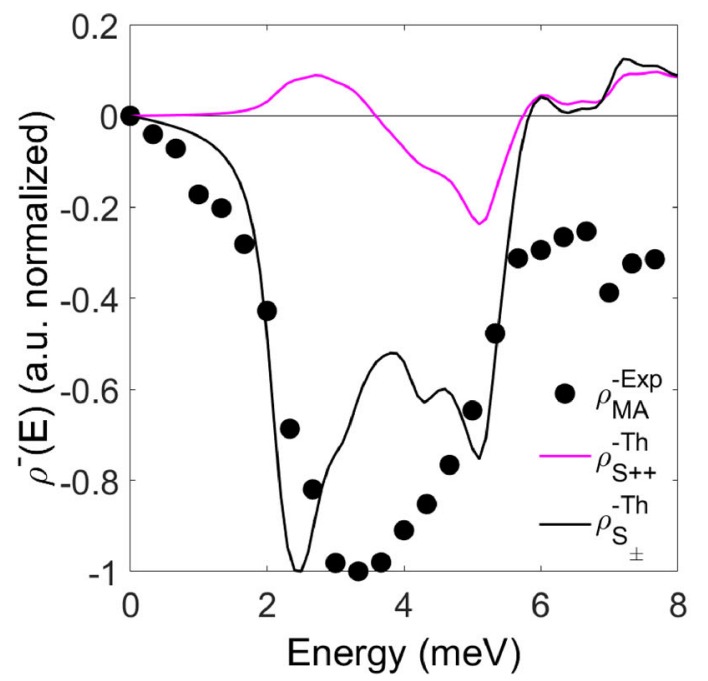

around $\Gamma$-point and two electron pockets $e_{1}$ and $e_{2}$ around $X$ point. The hole pockets around $\Gamma$-point on the FS revealed by spectroscopic imaging STM (SI-STM) ${ }^{18}$ and confirmed by angle resolved photoemission spectroscopy (ARPES) ${ }^{29,30}$, are much smaller as compared to most other Fe-based superconductors. Local density approximation (LDA) and dynamical mean field theory (DMFT) calculations have attributed the small size of hole pocket to stronger electron-electron correlation in this material. The superconducting energy-gaps $\Delta_{\mathbf{k}}^{a}$ are substantially anisotro$\mathrm{pic}^{18}$. Theoretically, in the case of $\Delta_{\mathbf{k}}^{a}$ with $\mathrm{s}_{ \pm}$symmetry, if both electron-like and hole-like pockets are present ${ }^{31,32}$, the pairing arises from spin-fluctuations which are enhanced by nesting between the electron-like and hole-like pockets. But the presence of three hole pockets, combined with relatively weak spin fluctuations ${ }^{33}$, allow for several possible competing ground states in the presence of repulsive interactions. In ref. ${ }^{34}$, it was pointed out that, under these conditions, several s-wave channels are nearly degenerate. These channels include the $s_{ \pm}$state where the signs on all hole pockets are the same $e^{35,36}$ and opposite to the signs on the electron bands, so-called "orbital antiphase state" that occurs when the interaction is diagonal in orbital space ${ }^{24}$, and a distinct sign structure obtained when vertex corrections were included $^{36}$. Reference 37 considered the question of whether these various proposed phases could be distinguished using HAEM based on Eq. (3) and concluded that it would be challenging.

Here we examine the relative signs of $\Delta_{\mathbf{k}}^{a}$ in LiFeAs by using MAHAEM. Figure 3a shows the typical cleaved surface of LiFeAs. The scattering sites used in our analysis are Fe-atom vacancies which are non-magnetic (Supplementary Fig. S3). The theoretical simulations for LiFeAs were performed from the experimentally fitted tight binding model ${ }^{27}$ and anisotropic gap magnitude structure ${ }^{18,30}$. At wavevectors corresponding to electron-hole scattering in q-space, a "horn-shaped" feature in $g(\mathbf{q}, E)$ appears within which we focus on an exemplary scattering vector $\mathbf{q}_{\mathrm{eh}}$ indicated by a dashed arrow in Fig. 3b. Figure $3 \mathrm{~d}$ then shows the theoretical, single-atom $\rho^{-T h}(\mathbf{q}, E)$ integrated for the $\mathbf{q}$ in the brown oval in Fig. $3 c$ for $s_{ \pm}$and $s_{++}$gaps, where sign of the gap was imposed by hand. The sign of $\rho_{s \pm}^{-T h}$ does not change for the energy values within the superconducting gap and its amplitude peaks at the energy $E \approx \Delta^{\mathrm{e}_{1}} \Delta^{\mathrm{h}_{1}}$, both characteristics of a sign changing gap ${ }^{37}$; contrariwise $\rho_{s_{++}}^{- \text {Th }}$ changes sign indicative of same sign energy gaps throughout.

For comparison, differential conductance $g(\mathbf{r}, E)$ imaging of LiFeAs is performed at $T=1.2 \mathrm{~K}$. The typical $g(E)$ spectrum consists of two gaps corresponding to $\Delta_{1}=5.3 \mathrm{meV}$ and $\Delta_{2}=2.6 \mathrm{meV}$. The measured $g(\mathbf{q}, E)$ are shown in Fig. $4 a$ and the feature at $\mathbf{q}_{\mathrm{eh}}$ expected from the theoretical model in Fig. $3 \mathrm{c}$ is indicated by a circle. We evaluate $\rho_{\mathrm{MA}}^{-}(\mathbf{q}, E)$ from Eq. (9) for $N=100$ atomic scale Fe-atom vacancy sites (Supplementary Note 4). The resulting

Fig. 4 LiFeAs superconducting energy-gap symmetry from MAHAEM. a The measured $|g(\mathbf{q})|$ pattern recorded in the FOV with multiple atomic scattering sites. The hole-like to electron-like scattering as predicted in Fig. $3 c$ is detected clearly and indicated by a brown circle at the same location as $3 c$. The features inside the circle do not appear identical because the intensity in real experimental data falls off at higher $|\mathbf{q}|$, while the theoretical simulation is replicated about the q-space Brillouin zone boundary with the same intensity, creating a non-physical equal intensity reflected feature. A Gaussian mask of $\sigma=0.68 \AA^{-1}=0.8 \pi / a$ is used to suppress the $|g(\mathbf{q})|$ data in the $|\mathbf{q}| \approx 0$ core region, to allow clearer presentation of the high-|q|- under study. Much of this $|\mathbf{q}| \approx 0$ signal intensity is believed to emanate from long-range disorder and is, moreover, unrelated to the scientific objectives of this paper. $\mathbf{b}$ The measured $\rho_{\mathrm{MA}}^{-}(\mathbf{q}, E=3.33 \mathrm{meV})$ using Eq. (9); it is typical of all $\rho_{\mathrm{MA}}^{-}(\mathbf{q}, E)$ between 1 and $6 \mathrm{meV}$. The circle indicates the hole-like to electron-like scattering in a. We integrate the $\rho_{\mathrm{MA}}^{-}(\mathbf{q}, E)$ over the range of $\mathbf{q}$ within this region. The dashed lines are guide to eye to a feature which is consistent with intra $h_{3}$ scattering. Again, a Gaussian mask of $\sigma=0.68 \AA^{-1}=0.8 \pi / a$ is used to suppress the intense core emanating from long range disorder, to allow clearer presentation of the $\rho_{\mathrm{MA}}^{-}(\mathbf{q}, E)$ information at high-|q| . This suppressed area corresponds to $\sim 18 \%$ of the total area of the first $\mathbf{q}$ space Brillouin zone, and the unprocessed $\rho_{M A}^{-}(\mathbf{q}, E)$ data for this figure are provided in its entirety at Supplementary Fig. S6a. c The resulting $\rho_{\mathrm{MA}}^{-}(E)$ calculated by summing over the oval enclosed region in $\mathbf{b}$ (black dots), and the theory curves (solid) for $s_{ \pm}$(black) and $s_{++}$(pink) overlaid. Clearly, this demonstrates using MAHAEM that the superconducting energy gap symmetry of LiFeAs is $s_{ \pm}$ (black). 
image $\rho_{\mathrm{MA}}^{-}(\mathbf{q}, E)$ at a representative subgap energy $E=3.3 \mathrm{meV}$ is shown in Fig. 4b.

Of note in Fig. $4 b$ is the variety of structures at $|\mathbf{q}| \ll\left|\mathbf{q}_{\mathrm{eh}}\right|$, which are challenging to understand. The thin outer blue ring (indicated by dashed light blue curve as guide-to-eye) is located at a radius in $\mathbf{q}$-space that corresponds well to the expected intraband scattering within pocket $h_{3}$. Furthermore, much of the q-space within this ring is blue and of rather high intensity for $1 \mathrm{meV}<|E|<6 \mathrm{meV}$ (Supplementary Fig. S6a shows dashed contours for various possible inter-hole-band scatterings overlaid on the unprocessed $\rho^{-}(\mathbf{q}, E)$ ). The blue color, indicating signpreserving scattering, is consistent with the conventional $\mathrm{s}_{ \pm}$ picture within a HAEM scenario, but the high intensity is not. As discussed in Supplementary Note 5 there are several possible explanations of these low $|\mathbf{q}|$ phenomena, including strong scattering, quasiparticle bound states, and antiphase holepocket gaps.

Nevertheless, when the high $|\mathbf{q}|$ scattering between hole-like and electron-like pockets (Fig. $3 \mathrm{~b}, \mathrm{c}$ ) is integrated within the $\mathbf{q}$ space region shown by a brown circle on the $\rho_{\mathrm{MA}}^{-}(\mathbf{q}, E)$ of Fig. $4 \mathrm{a}$, it yields $\rho_{\mathrm{MA}}^{-}(E)$ as plotted in Fig. 4c. The theoretically predicted $\rho^{-}(E)$ curves are overlaid for comparison. It is clear that the experimental $\rho_{\mathrm{MA}}^{-}(E)$ is consistent with the $\rho_{ \pm}^{-\mathrm{Th}}(E)$ theory because it does not change sign and exhibits a peak at $E \approx 3.7 \mathrm{meV} \approx \sqrt{\Delta_{1} \Delta_{2}}$. In this way, the MAHAEM technique efficiently demonstrates that $\Delta_{\mathbf{k}}^{a}$ changes sign between electronlike and hole-like bands of LiFeAs.

\section{Conclusions}

We report development and demonstration of an improved approach for signed $\Delta_{\mathbf{k}}^{a}$ determination (Eq. (9)), but now for use with multiple impurity atoms or scattering centers. This MAHAEM technique for measuring $\rho^{-}(\mathbf{q}, E)$ is based on a combination of the Fourier shift theorem and high-precision registry of scatterer locations. It extends the original HAEM approach ${ }^{4}$ to more disordered superconductors (Figs. 2a, 3a), enables its application to far larger fields of view thereby enhancing $\mathbf{q}$-space resolution (Fig. 4b), and greatly increases signal-to-noise ratios (Figs. 1d, 4b) by suppressing phase randomization in multi-atom scattering interference. Overall, MAHAEM now represents a powerful and general technique for $\Delta_{\mathbf{k}}^{a}$ determination in complex superconductors.

\section{METHODS}

Sample growth and preparation

FeSe samples with $T_{\mathrm{c}} \approx 8.7 \mathrm{~K}$ were prepared using $\mathrm{KCl}_{3} / \mathrm{AlCl}_{3}$ chemicalvapor transport and LiFeAs samples with $T_{\mathrm{c}} \approx 15 \mathrm{~K}$ were grown using LiAs flux method. The highly reactive LiFeAs samples are prepared in a dry nitrogen atmosphere in a glove box.

\section{SI-STM measurements and analysis}

All samples are cleaved in situ in our ultra-high cryogenic vacuum STM at low temperature. The $g(\mathbf{r}, E)$ data were acquired with a ${ }^{3} \mathrm{He}$-refrigeratorequipped STM. The picometer level atomic registration was performed before applying the HAEM technique as described in full detail in the Supplementary Note 2. Full details of the multi-atom HAEM analysis are presented in detail in Supplementary Note 3. Theoretical predictions for $\rho^{-}(E)$ curves were performed using the T-matrix formalism with energy gap on each band and normal state tight binding parameters fitted to experiments.

\section{DATA AVAILABILITY}

The datasets generated and/or analyzed during this study are available to qualified requestors from the corresponding author.

\section{CODE AVAILABILITY}

The simulation code for Fig. 1 is provided as Supplemental material. All the other codes used during the current study are available to qualified requestors from the corresponding author.

Received: 11 May 2020; Accepted: 26 November 2020; Published online: 11 January 2021

\section{REFERENCES}

1. Norman, M. R. The challenge of unconventional superconductivity. Science $\mathbf{3 3 2}$, 196-200 (2011).

2. Van Harlingen, D. J. Phase-sensitive tests of the symmetry of the pairing state in the high-temperature superconductors-evidence for $d_{x^{2}-y^{2}}$ symmetry. Rev. Mod. Phys. 67, 515 (1995).

3. Tsuei, C. C. \& Kirtley, J. R. Pairing symmetry in cuprate superconductors. Rev. Mod. Phys. 72, 969 (2000).

4. Hirschfeld, P. J., Altenfeld, D., Eremin, I. \& Mazin, I. I. Robust determination of the superconducting gap sign structure via quasiparticle interference. Phys. Rev. B 92, 184513 (2015).

5. Wang, Q. H. \& Lee, D. H. Quasiparticle scattering interference in high-temperature superconductors. Phys. Rev. B 67, 020511(R) (2003).

6. Sprau, P. O. et al. Discovery of orbital-selective Cooper pairing in FeSe. Science 357, 75-80 (2017).

7. $\mathrm{Du}, \mathrm{Z}$. et al. Sign reversal of the order parameter in $\left(\mathrm{Li}_{1-\mathrm{x}} \mathrm{Fe}_{\mathrm{x}}\right) \mathrm{OHFe}_{1_{-y}} \mathrm{Zn}_{\mathrm{y}} \mathrm{Se}$. Nat. Phys. 14, 134-139 (2017).

8. Chi, S. et al. Determination of the superconducting order parameter from defect bound state quasiparticle interference. Preprint at https://arxiv.org/abs/ 1710.09089 (2017).

9. Chen, $M$. et al. Direct visualization of sign-reversal $s_{ \pm}$-superconducting gaps in $\mathrm{FeTe}_{0.55} \mathrm{Se}_{0.45}$. Phys. Rev. B 99, 014507 (2019).

10. $\mathrm{Gu}, \mathrm{Q}$. et al. Directly visualizing the sign change of $d$-wave superconducting gap in $\mathrm{Bi}_{2} \mathrm{Sr}_{2} \mathrm{CaCu}_{2} \mathrm{O}_{8+\delta}$ by phase-referenced quasiparticle interference. Nat. Commun. 10, 1603 (2019).

11. Böker, J. et al. Phase-sensitive determination of nodal $d$-wave order parameter in single-band and multiband superconductors. Phys. Rev. B 101, 214505 (2020).

12. Cheung, S. C. et al. Dictionary learning in Fourier-transform scanning tunneling spectroscopy. Nat. Commun. 11, 1081 (2020).

13. Lawler, M. J. et al. Intra-unit-cell electronic nematicity of the high-Tc copper-oxide pseudogap states. Nature 466, 347-351 (2010).

14. Fujita, K. et al. Direct phase-sensitive identification of a d-form factor density wave in underdoped cuprates. Proc. Natl Acad. Sci. USA 111, E3026-E3032 (2014).

15. Hamidian, M. H. et al. Picometer registration of zinc impurity states in $\mathrm{Bi}_{2} \mathrm{Sr}_{2} \mathrm{Ca}$ $\mathrm{Cu}_{2} \mathrm{O}_{8+\delta}$ for phase determination in intra-unit-cell Fourier transform STM. New J. Phys. 14, 053017 (2012).

16. Hoffman, J. E. et al. Imaging Quasiparticle Interference in $\mathrm{Bi}_{2} \mathrm{Sr}_{2} \mathrm{CaCu}_{2} \mathrm{O}_{8+\delta}$. Science 297, 1148-1151 (2002).

17. Hanaguri, T. et al. Quasiparticle interference and superconducting gap in $\mathrm{Ca}_{2-x} \mathrm{Na}_{x} \mathrm{CuO}_{2} \mathrm{Cl}_{2}$. Nat. Phys. 3, 865-871 (2007).

18. Allan, M. P. et al. Anisotropic energy gaps of iron-based superconductivity from intraband quasiparticle interference in LiFeAs. Science 336, 563-567 (2012).

19. Allan, M. P. et al. Imaging Cooper pairing of heavy fermions in CeColn ${ }_{5}$. Nat. Phys. 9, 468-473 (2013).

20. Zhou, B. B. et al. Visualizing nodal heavy fermion superconductivity in CeColn ${ }_{5}$. Nat. Phys. 9, 474-479 (2013).

21. Du, Z. et al. Scrutinizing the double superconducting gaps and strong coupling pairing in $\left(\mathrm{Li}_{1-\mathrm{x}} \mathrm{Fe}_{\mathrm{x}}\right) \mathrm{OHFeSe.} \mathrm{Nat.} \mathrm{Commun.} \mathrm{7,} 10565$ (2016).

22. Hanaguri, $T$. et al. Two distinct superconducting pairing states divided by the nematic end point in $\mathrm{FeSe}_{1-x} \mathrm{~S}_{\mathrm{x}}$. Sci. Adv. 4, eaar6419 (2018).

23. Sharma, $\mathrm{R}$. et al. Momentum resolved superconducting energy gaps of $\mathrm{Sr}_{2} \mathrm{RuO}_{4}$ using quasiparticle interference. Proc. Natl Acad. Sci. USA 117, 5222-5227 (2020).

24. Lu, X., Fang, C., Tsai, W.-F., Jiang, Y. \& Hu, J. s-wave superconductivity with orbitaldependent sign change in checkerboard models of iron-based superconductors. Phys. Rev. B 85, 054505 (2012).

25. Yin, Z. P., Haule, K. \& Kotliar, G. Spin dynamics and orbital-antiphase pairing symmetry in iron-based superconductors. Nat. Phys. 10, 845-850 (2014).

26. Hirschfeld, P. J. Using gap symmetry and structure to reveal the pairing mechanism in Fe-based superconductors. C. R. Phys. 17, 197-231 (2016).

27. Eschrig, H. \& Koepernik, K. Tight-binding models for the iron-based superconductors. Phys. Rev. B 80, 104503 (2009).

28. Wang, Y. et al. Superconducting gap in LiFeAs from three-dimensional spinfluctuation pairing calculations. Phys. Rev. B 88, 174516 (2013). 
29. Borisenko, S. V. et al. One-sign order parameter in iron based superconductor. Symmetry 4, 251-264 (2012).

30. Umezawa, K. et al. Unconventional anisotropic s-wave superconducting gaps of the LiFeAs iron-pnictide superconductor. Phys. Rev. Lett. 108, 037002 (2012)

31. Chubukov, A. Pairing mechanism in Fe-based superconductors. Annu. Rev. Condens. Matter Phys. 3, 57-92 (2012).

32. Maiti, S., Korshunov, M. M., Maier, T. A., Hirschfeld, P. J. \& Chubukov, A. V. Evolution of the superconducting state of Fe-based compounds with doping. Phys. Rev. Lett. 107, 147002 (2011).

33. Qureshi, N. et al. Fine structure of the incommensurate antiferromagnetic fluctuations in single-crystalline LiFeAs studied by inelastic neutron scattering. Phys. Rev. B 90, 144503 (2014).

34. Ahn, F. et al. Superconductivity from repulsion in LiFeAs: novel s-wave symmetry and potential time-reversal symmetry breaking. Phys. Rev. B 89, 144513 (2014).

35. Platt, C., Thomale, R. \& Hanke, W. Superconducting state of the iron pnictide LiFeAs: a combined density-functional and functional-renormalization-group study. Phys. Rev. B 84, 235121 (2011).

36. Saito, T. et al. Reproduction of experimental gap structure in LiFeAs based on orbital-spin fluctuation theory: $s_{++}-$wave, $s_{ \pm}$-wave, and hole- $s_{ \pm}-$wave states. Phys. Rev. B 90, 035104 (2014).

37. Altenfeld, D., Hirschfeld, P. J. H., Mazin, I. I. \& Eremin, I. Detecting sign-changing superconducting gap in LiFeAs using quasiparticle interference. Phys. Rev. B 97 054519 (2018).

38. Rhodes, L. C. et al. Revealing the single electron pocket of FeSe in a single orthorhombic domain. Phys. Rev. B 101, 235128 (2020).

\section{ACKNOWLEDGEMENTS}

Work done by P.C.C. and A.E.B. was supported by the U.S. Department of Energy, Office of Basic Energy Science, Division of Materials Sciences and Engineering and was performed at the Ames Laboratory. Ames Laboratory is operated for the U.S. Department of Energy by lowa State University under Contract No. DE-AC0207CH11358. R.S. acknowledges support from Cornell Center for Materials Research with funding from the NSF MRSEC program (DMR-1719875). The authors are thankful to M.A. Müller for the discussion of the QPI results in LiFeAs. P.J.H. and M.A.S. acknowledge support from NSF-DMR-1849751; H.E. acknowledges Grant-in-Aid for Scientific Research on Innovative Areas "Quantum Liquid Crystals" (KAKENHI Grant No. JP19H05823) from JSPS of Japan. J.C.S.D. acknowledges support from the Moore Foundation's EPiQS Initiative through Grant GBMF9457, from the Royal Society through Award R64897, from Science Foundation Ireland under Award SFI 17/RP/ 5445, and from the European Research Council (ERC) under Award DLV-788932.

\section{AUTHOR CONTRIBUTIONS}

R.S., A.Kr., and M.A.S. contributed to this project equally. P.O.S., R.S., P.J.H., and J.C.S.D designed the project. P.O.S. and M.A.S. developed the phase-resolved multi-atom averaging method; M.P.A., A.Ko., and P.O.S. carried out the experiments; R.S. and P.O.S. carried out the data analysis; A.Kr., M.A.S., J.B., P.J.H., and I.E. carried out the theoretical analysis. P.C.C. and A.E.B. synthesized single-crystalline FeSe samples; H.E. synthesized single-crystalline LiFeAs samples. J.C.S.D. and P.J.H. supervised the investigation and wrote the paper with key contributions from P.O.S., R.S., M.A.S., and A.Kr. The manuscript reflects the contributions of all authors.

\section{COMPETING INTERESTS}

The authors declare no competing interests.

\section{ADDITIONAL INFORMATION}

Supplementary information is available for this paper at https://doi.org/10.1038/ s41535-020-00303-4.

Correspondence and requests for materials should be addressed to J.C.S.D.

Reprints and permission information is available at http://www.nature.com/ reprints

Publisher's note Springer Nature remains neutral with regard to jurisdictional claims in published maps and institutional affiliations.

(i) Open Access This article is licensed under a Creative Commons Attribution 4.0 International License, which permits use, sharing adaptation, distribution and reproduction in any medium or format, as long as you give appropriate credit to the original author(s) and the source, provide a link to the Creative Commons license, and indicate if changes were made. The images or other third party material in this article are included in the article's Creative Commons license, unless indicated otherwise in a credit line to the material. If material is not included in the article's Creative Commons license and your intended use is not permitted by statutory regulation or exceeds the permitted use, you will need to obtain permission directly from the copyright holder. To view a copy of this license, visit http://creativecommons. org/licenses/by/4.0/.

(c) Crown 2021 